\title{
CRESCIMENTO SOMÁTICO E RELATIVO DE PORTUNUS SPINICARPUS (STIMPSON, 1971) (CRUSTACEA PORTUNIDAE) NO LITORAL NORTE DE SANTA CATARINA, BRASIL
}

\author{
CYNTHIA Y. OGAWA ${ }^{1} \&$ FERNANDO D'INCAO ${ }^{2}$ \\ Universidade Federal do Rio Grande, Instituto de Oceanografia, Av. Itália km 8, C. Postal 474, 96201-900, Rio Grande, RS, Brasil. \\ cynthiaogawa@yahoo.com.br, docdincao@furg.br
}

\begin{abstract}
SOMATIC AND RELATIVE GROWTH OF PORTUNUS SPINICARPUS (STIMPSON, 1971) (CRUSTACEA PORTUNIDAE) AT SANTA CATARINA NORTHERN'S COAST, BRAZIL

Somatic and relative growth of Portunus spinicarpus were studied in Santa Catarina, Brazil. Samples were obtained through the Project Camarões da Baía da Babitonga (CEPSUL/IBAMA, UNIVALI, UNIVILLE, FURG) at a depth range of 10-100 m in the coast area that corresponds to Sao Francisco do Sul and Joinville, during eight cruises performed from July, 2004 to July, 2006. Carapace width varied between 16.0 and $92.0 \mathrm{~mm}$ and 18.0 and $103.0 \mathrm{~mm}$ for males and females, respectively. Females coefficient of growth $(k=0.0055 /$ day $)$ did not differ significantly from males $(k=0.0052 /$ day), however females reached larger asymptotic size $\left(\mathrm{LC}_{\infty}=103.28\right)$ than males $\left(\mathrm{LC}_{\infty}=92.00\right)$. Width-weight relationship for females was isometric while for males two patterns were observed: a negative allometric for individuals smaller than $60.0 \mathrm{~mm}$ and a positive allometric for individuals with CW equal to $60.0 \mathrm{~mm}$ or bigger. That relationship was significantly different between sexes. Longevity was 2,4 years for males and 2,3 years for females. Overall, the results demonstrate that relative growth in $P$. spinicarpus correspond to that in other species of Decapods, except that females are bigger than males and that should be further examined.
\end{abstract}

KEY WORDS: swimming crab, Portunidae, growth, relative growth, Santa Catarina

\section{RESUMO}

O crescimento somático e relativo de Portunus spinicarpus foi estudado no litoral de Santa Catarina. As amostras foram obtidas como parte do Projeto Camarões da Baía da Babitonga (CEPSUL/IBAMA, UNIVALI, UNIVILLE, FURG) em uma área cuja profundidade varia de 10 a $100 \mathrm{~m}$ e corresponde a São Francisco do Sul e Joinville. Foram realizados oito cruzeiros entre julho de 2004 a julho de 2006. A largura de carapaça variou entre 16,0 e 92,0 mm (machos) e 18,0 e 103,0 mm (fêmeas). A taxa de crescimento das fêmeas $(k=0,0055 /$ dia) não diferiu significativamente dos machos ( $k=0,0052 /$ dia $)$, no entanto as fêmeas atingiram tamanho maior $(\mathrm{LC} \infty=103,28)$ do que os machos $(\mathrm{LC} \infty=92,00)$. A relação peso total e largura de carapaça para fêmeas é isométrica enquanto nos machos é alométrica negativa para indivíduos menores que $60,0 \mathrm{~mm}$ passando a alométrica positiva a partir dessa classe de tamanho. Essa relação mostrou-se significativamente diferente entre os sexos. A longevidade dos machos foi estimada em 2,4 anos e para fêmeas em 2,3 anos. Em geral, $P$. spinicarpus em Santa Catarina possui características similares ao de outros decápodes exceto que fêmeas apresentaram tamanho maior que machos e esta características deve ser melhor investigada.

PALAVRAS CHAVE: siri, Portunidae, crescimento, crescimento relativo, Santa Catarina

\section{INTRODUÇÃO}

Portunus spinicarpus (Stimpson, 1871) ocorre ao longo do Atlântico ocidental, desde os Estados Unidos (Carolinas do Norte e do Sul e Flórida), Golfo do México, Antilhas, Colômbia, Venezuela, Guianas até o Brasil (do Amapá até o Rio Grande do Sul), habitando desde águas rasas até $550 \mathrm{~m}$, e em substratos de areia, cascalho, conchas quebradas, corais e também em lama (Melo, 1996).

Hartnoll (1982) definiu o crescimento como o aumento do comprimento, volume, peso úmido ou peso seco com o passar do tempo. De acordo com o mesmo autor, a presença de um exoesqueleto pouco extensível faz com que o crescimento em crustáceos seja essencialmente um processo descontínuo, onde há uma sucessão de mudas ou ecdises separadas por um período de intermuda. A melhor forma de descrever o crescimento nesse grupo é pela observação dos padrões de muda (Josileen \& Menon, 2005).
A maioria das tentativas de se determinar a idade e descrever o crescimento de espécies do gênero Portunus tem empregado estudos subjetivos de progressão modal em freqüência de tamanho ao longo do tempo (de Lestang, 2003b; Potter et al., 1983; Sukumuran \& Neelakantan, 1997; Sumpton et al., 1994). A curva de von Bertalanffy derivada para machos e fêmeas nas análises feitas por Sumpton et al (1994) e Sukumuran \& Neelakantan (1997) não continha um termo em suas equações de crescimento que levasse em conta qualquer variação sazonal na taxa de crescimento dessas espécies. Da mesma maneira, não foi constatado diferença no crescimento entre machos e fêmeas de $P$. pelagicus (de Lestang et al., 2003a).

Ao longo do crescimento é comum que certas dimensões cresçam a taxas diferentes de outras, de modo a mostrar uma diferença na proporção com o tamanho: o crescimento relativo. Os Crustacea têm se mostrado, particularmente, adequados para esse tipo de estudo (Hartnol, 1978). O crescimento relativo está intimamente relacionado com a maturação 
gonadal e o período de muda. Neste sentido, relações entre o comprimento ou largura da carapaça têm sido usadas como importantes ferramentas para descrever essas mudanças (Hartnol, 1982)

Características de tendências alométricas para grandes grupos taxonômicos (por exemplo, gênero e famílias), usualmente, foram estabelecidas pela analise de apenas algumas espécies dentro do grupo. Entretanto, há uma considerável variação nos padrões do crescimento relativo dentro de um grupo para o qual uma "tendência" foi postulada. Tendências alométricas somente podem ser compreendidas e deveriam apenas ser postuladas dentro de um contexto filogenético (Corbi-Côrrea \& Fransozo, 2002).

Para Portunus spinicarpus na região de Ubatuba, São Paulo, Corbi-Côrrea \& Fransozo (2002) estabeleceram as relações alométricas entre largura de carapaça, comprimento da carapaça, largura do abdômen de fêmeas, comprimento da quela, comprimento do carpo mais o espinho. A relação do peso do corpo em função da largura de carapaça do siri candeia $P$. spinimanus foi determinada por Santos et al (1995) para a mesma região. Ávila \& Branco (1996) estimaram a relação do peso em função do comprimento e da largura de carapaça para Arenaeus cribrarius em Santa Catarina.
O presente trabalho teve por objetivo estimar a curva de crescimento para a espécie segundo von Bertalanffy, bem como crescimento relativo entre largura de carapaça e peso para machos e fêmeas.

\section{MATERIAL E MÉTODOS Área de Estudo}

A área de estudo corresponde à região marinha adjacente a Baía da Babitonga, próximo às cidades de São Francisco do Sul e Joinville, no litoral norte do Estado de Santa Catarina. O quadrante formado

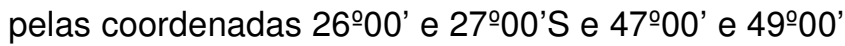
W delimita a zona onde foram estabelecidas as 19 estações de arrasto (Figura 1).

Hidrologicamente, todo o litoral sul do Brasil, em profundidade maior que vinte metros, sofre efeitos da intrusão de uma massa de água denominada Água Central do Atlântico Sul (ACAS), por baixo das águas costeiras dominadas pela Corrente do Brasil (Klein et al., 2001). Além da ACAS também se observa na região a influência da Água Tropical (AT) e da Água Costeira (AC). De acordo com Castro \& Miranda (1987) tais massas de água são bem distintas entre si quanto à temperatura, salinidade e riqueza em nutrientes.

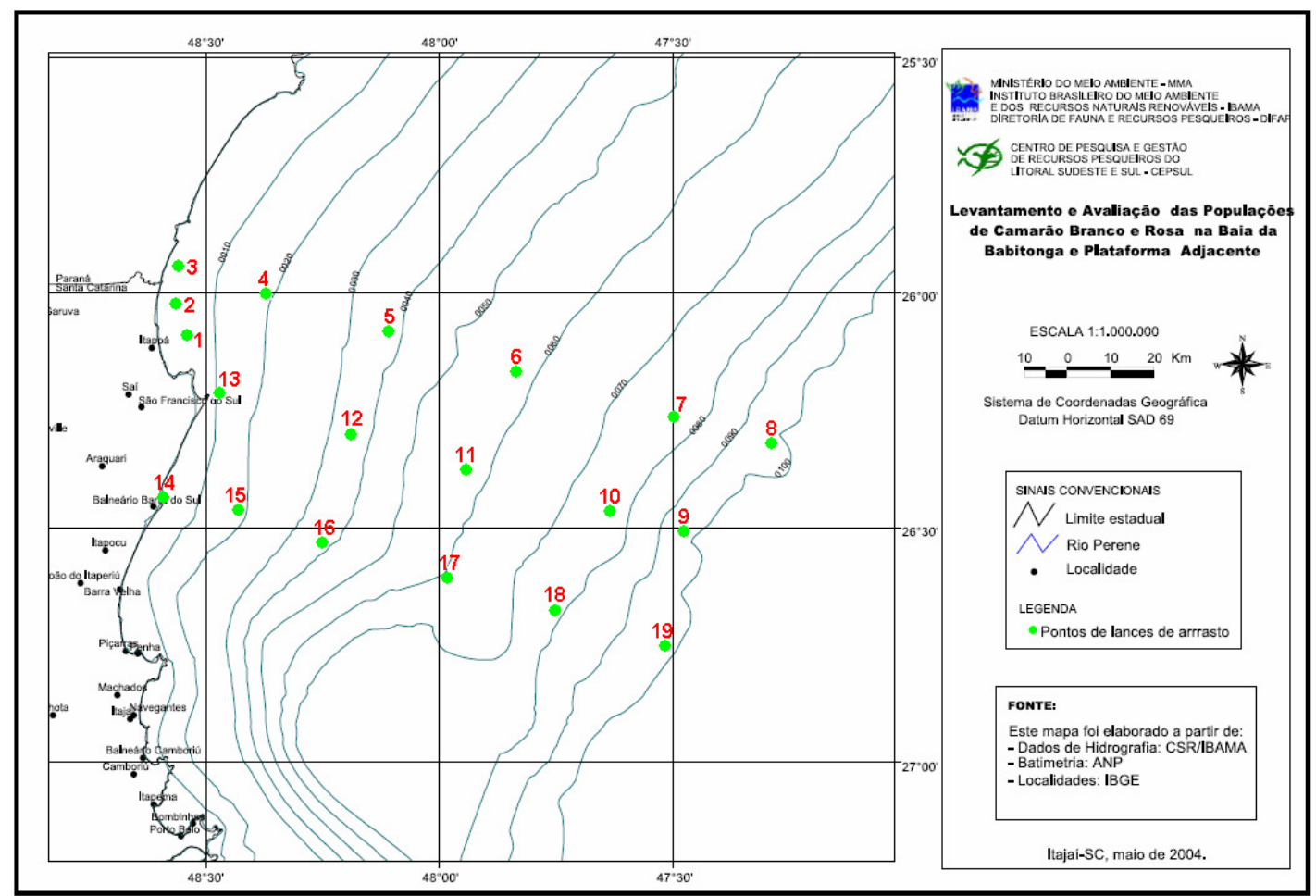

Figura 1. Carta náutica mostrando em detalhe a área de estudo, situada na frente da Baía da Babitonga, São Francisco do Sul, Litoral Norte de Santa Catarina, Brasil. Os pontos numerados de 1 a 19 indicam as estações de coleta. 


\section{Metodologia de Coleta}

As coletas foram realizadas como parte do "Projeto Camarões da Baía de Babitonga - CAMBA" executado pelo CEPSUL/IBAMA (Centro de Pesquisa do Sul - Itajaí) em conjunto com a Universidade da Região de Joinville - UNIVILLE, Universidade Vale do Itajaí - UNIVALI e o Laboratório de Crustáceos Decápodes - LCD da Universidade Federal do Rio Grande - FURG. Ao todo foram realizados oito cruzeiros a bordo do N.Oc. Soloncy Moura: junho-julho/04, agostosetembro/04, janeiro/05, março/05, outubronovembro/05, março/06, abril-maio/06 e julho/06.

Em cada cruzeiro realizaram-se arrastos com rede camaroneira de portas com as seguintes características: $22 \mathrm{~m}$ de abertura horizontal não operacional, malhas do corpo com $40 \mathrm{~mm}$ (entre-nós opostos, malha esticada), malhas da região do saco medindo $20 \mathrm{~mm}$ (entre-nós opostos malha esticada). A rede foi operada a uma velocidade média de 3 nós durante $30 \mathrm{~min}$.

Foram estabelecidas 19 estações de arrasto, distribuídas ao longo de três transectas perpendiculares a linha da costa de acordo com as profundidades de 10, 20, 40, 60, 80 e $100 \mathrm{~m}$ (Figura 1).

As amostras obtidas em cada arrasto foram triadas a bordo, de modo a separar grandes grupos (ex. Crustáceos, peixes, etc.), acondicionadas em sacos plásticos, etiquetadas (número do lance) e congeladas. A identificação das espécies foi feita no laboratório do CEPSUL, em Itajaí. Os espécimes coletados de Portunus spinicarpus foram separados por sexo, pesados (balança analítica - 0,01 g) e tiveram a largura da carapaça medida entre as extremidades dos espinhos utilizando um com paquímetro $(0,1 \mathrm{~mm})$.

Os parâmetros do modelo de crescimento de von Bertalanffy foram estimados a partir de uma análise de progressão modal, conforme metodologia detalhada por Fonseca (1998).

Para análise modal os dados foram agrupados em classes de tamanho de 1,0 mm. As modas foram calculadas com software de ajuste Peak Fit 4.0, que ajusta as freqüências observadas a curvas normais, de maneira que os valores da média e da moda das normais ajustados são os mesmos.

Para a detecção e o cálculo dos valores centrais dos picos, foi utilizada a opção "Automatic peak detection and fitting (I)", escolhendo o ajuste a uma curva de Gauss (normal). Os picos sobrepostos foram excluídos, não sendo interpretados como grupos etários verdadeiros. Um resumo estatístico de cada um dos ajustes foi obtido através da saída numérica (Numeric) sendo o coeficiente de determinação (R2), os graus de liberdade (GL) e o valor de $\mathrm{F}$ crítico calculado. Um ajuste com valor de $F$ acima do $F$ crítico significa sua aceitação para análise.

As modas foram plotadas em um gráfico de dispersão contra o tempo, para acompanhar as coortes. Os parâmetros de crescimento foram calculados para as diferentes coortes utilizando-se a ferramenta solver do programa Excel (Office XP). Esta ferramenta utiliza os valores "sementes" para minimizar as somas dos resíduos entre as larguras de carapaça observadas e aquelas calculadas pelo modelo de Bertalanffy, variando os parâmetros ( $\mathrm{k}$ e $\left.t_{0}\right)$ da equação. Como parâmetro $\left(L_{\infty}\right)$ foi utilizado o maior valor de largura de carapaça observado para machos e fêmeas, separadamente. O valor de LC $_{\infty}$ foi fixado por ter apresentado o melhor ajuste para os dados.

A seleção das coortes levou em consideração a longevidade, coeficiente de crescimento e coeficiente de determinação $\left(R^{2}\right)$ alto.

Após a seleção das modas, realizou-se a correção das idades em dias, baseadas nos intervalos de tempo entre os cruzeiros e no valor de $t_{0}$ encontrado. As larguras de carapaça modais e as idades corrigidas foram utilizadas para o cálculo de uma curva de crescimento média, através da equação de von Bertalanffy, como segue:

$$
L C t=L C_{\infty}\left[1-e^{-k(t-t 0)}\right]
$$

Onde:

$\mathrm{LCt}=$ largura total de carapaça no tempo $\mathrm{t}$

$\mathrm{k}=$ constante de crescimento

$\mathrm{t}_{0}=$ parâmetro de ajuste, idade do indivíduo quando seu tamanho é igual a zero 
Diferenças no crescimento entre machos e fêmeas foram testadas por um teste $F$ (Cerrato, 1990).

A longevidade foi calculada com a equação inversa de von Bertalanffy (1938), com uma adaptação proposta por D'Incao \& Fonseca (1999), considerando $\mathrm{t}_{0}=0, \mathrm{LC}_{0}=1,3 \mathrm{~mm}$ e $\mathrm{LCi} / \mathrm{LC}_{\infty}=0,99$. O tamanho LCO foi estabelecido com base nos dados fornecidos por Bookhout \& Costlow (1974). Sendo a equação:

$$
\text { Tmáx }=(0-1 / k) \operatorname{Ln}\left(1-\mathrm{LCi}^{-} \mathrm{LC}_{\infty}\right)
$$

Onde:

Tmáx = longevidade

Para relação entre a largura da carapaça e o peso dos indivíduos amostrados, todos os indivíduos que apresentavam danos na carapaça ou perda de apêndices foram retirados da análise. Após transformação logarítmica dos dados empíricos e constatada a relação linear entre as variáveis envolvidas, resultou na expressão:

$$
\operatorname{LnP}=\mathrm{Lna}+\mathrm{bLnLC}
$$

Onde:

$\mathrm{P}=$ peso

LC =largura de carapaça

$a=$ fator de condição

$\mathrm{b}=$ declividade da reta

Os valores de "a" e "b" foram estimados pelo método dos mínimos quadrados aplicado à relação linear e estimado o coeficiente de correlação linear de Pearson ( $r$ ) para a expressão no programa Excel
2003. Segue-se com a interpretação do parâmetro "b" conforme o proposto por King (2003). Segundo este autor o parâmetro "b" indica se a relação entre - peso e a largura de carapaça é alométrica ou isométrica. Valores de "b" iguais a 3 indicam isometria, enquanto valores menores do que 3 são alométricos negativos (indivíduos crescem mais em largura que em peso) e valores maiores que 3 são alométricos positivos (indivíduos crescem mais em peso que em largura).

A relação entre peso e largura da carapaça foi estimada separadamente para machos e fêmeas ao longo do período de estudo. A comparação desta relação entre sexos foi verificada através de uma Análise de Covariância (ANCOVA).

\section{RESULTADOS}

Foram capturados 6613 Portunus spinicarpus em Santa Catarina, dos quais 3303 eram machos e 3313 fêmeas, sendo 327 ovígeras. Os indivíduos capturados apresentaram largura de carapaça variando de 16,0 a $92,0 \mathrm{~mm}$ (Média $\mathrm{LC}=41,17$ ) para machos e de 18,0 a 103,0 mm (Média ${ }_{L C}=43,14$ ) para fêmeas e de 27,3 a 95,0 mm (Média ${ }_{\mathrm{LC}}=58,25$ ) para fêmeas ovígeras.

Constatou-se que as distribuições de freqüência de largura de carapaça apresentaram comportamento polimodal, o que reflete as diferentes coortes que se incorporam às populações ao longo do tempo (Figura 2). 

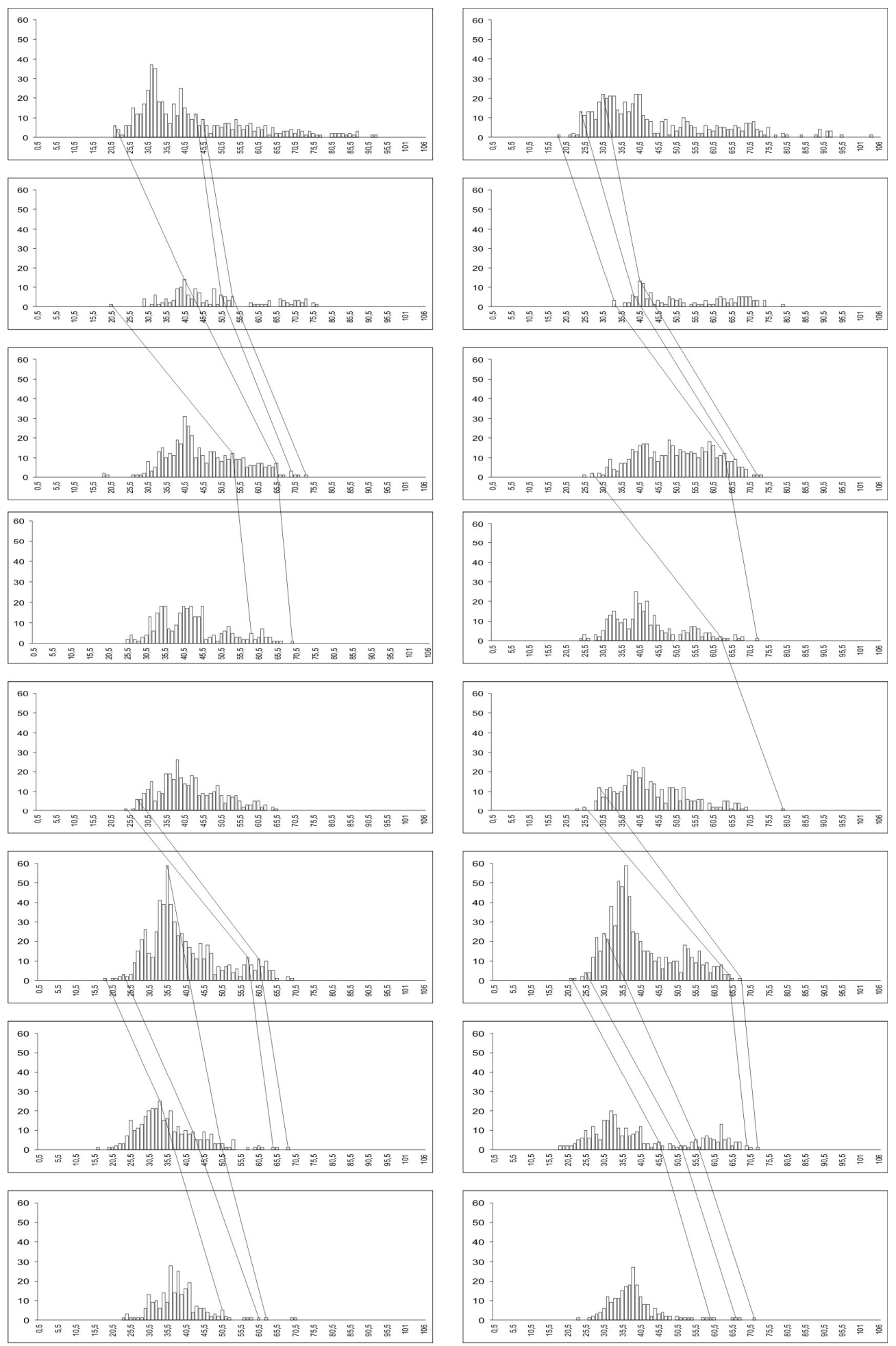

Figura 2. Análise da progressão modal de machos (histogramas da esquerda) e de fêmeas (histogramas da direita) de Potunus spinicarpus coletados no Litoral Norte de Santa Catarina, Brasil, durante o período de estudo. As linhas representam as coortes seguidas ao longo do tempo para descrever o crescimento individual.

Foram selecionadas 10 coortes de machos (Tabela 1) e 9 coortes de fêmeas (Tabela 2) para a análise das curvas de crescimento. Os pontos escolhidos para representar as coortes utilizadas nas análises de crescimento de machos e de fêmeas encontram-se na Figura 3. 
Tabela 1. Parâmetros de crescimento (largura de carapaça assintótica, $L C_{¥}$ e taxa de crescimento, K), longevidade máxima e ajuste das coortes de machos de Portunus spinicarpus coletados no Litoral Norte de Santa Catarina, durante o período de estudo.

\begin{tabular}{l|c|c|c|c}
\hline \multirow{2}{*}{ CoORTES } & \multirow{2}{*}{$\mathbf{L C}_{¥}(\mathbf{m m})$} & \multirow{2}{*}{$\mathbf{k}\left(\mathbf{d i a}^{-1}\right)$} & \multicolumn{2}{|c}{ Longevidade máxima } \\
\cline { 4 - 5 } & & & dias & anos \\
\hline Coorte 1 & 92,00 & 0,006 & 713,15 & 1,95 \\
Coorte 2 & 92,00 & 0,005 & 995,19 & 2,73 \\
Coorte 3 & 92,00 & 0,005 & 926,34 & 2,54 \\
Coorte 4 & 92,00 & 0,006 & 785,95 & 2,15 \\
Coorte 5 & 92,00 & 0,006 & 797,79 & 2,19 \\
Coorte 6 & 92,00 & 0,005 & 909,50 & 2,49 \\
Coorte 7 & 92,00 & 0,006 & 771,73 & 2,11 \\
Coorte 8 & 92,00 & 0,005 & 917,30 & 2,51 \\
Coorte 9 & 92,00 & 0,006 & 771,73 & 2,11 \\
Coorte 10 & 92,00 & 0,006 & 817,00 & 2,24 \\
\hline
\end{tabular}

Tabela 2. Parâmetros de crescimento (largura de carapaça assintótica, $L C_{¥}$ e taxa de crescimento, K), longevidade máxima e ajuste das coortes de fêmeas de Portunus spinicarpus coletados no Litoral Norte de Santa Catarina durante o período de estudo.

\begin{tabular}{l|c|c|c|c}
\hline \multirow{2}{*}{ COORTES } & \multirow{2}{*}{$\mathbf{L C} ¥ \mathbf{( m m})$} & \multirow{2}{*}{$\mathbf{k}\left(\mathbf{d i a}^{-1}\right)$} & \multicolumn{2}{|c}{ Longevidade máxima } \\
\cline { 3 - 5 } & & & $\mathbf{d i a s}$ & $\mathbf{a n o s}$ \\
\hline Coorte 1 & 103,28 & 0,005 & 864,13 & 2,37 \\
Coorte 2 & 103,28 & 0,006 & 789,00 & 2,16 \\
Coorte 3 & 103,28 & 0,006 & 716,92 & 1,96 \\
Coorte 4 & 103,28 & 0,005 & 890,67 & 2,44 \\
Coorte 5 & 103,28 & 0,005 & 918,85 & 2,52 \\
Coorte 6 & 103,28 & 0,005 & 886,68 & 2,43 \\
Coorte 7 & 103,28 & 0,005 & 917,44 & 2,51 \\
Coorte 8 & 103,28 & 0,006 & 717,76 & 1,97 \\
Coorte 9 & 103,28 & 0,007 & 683,23 & 1,87 \\
\hline
\end{tabular}



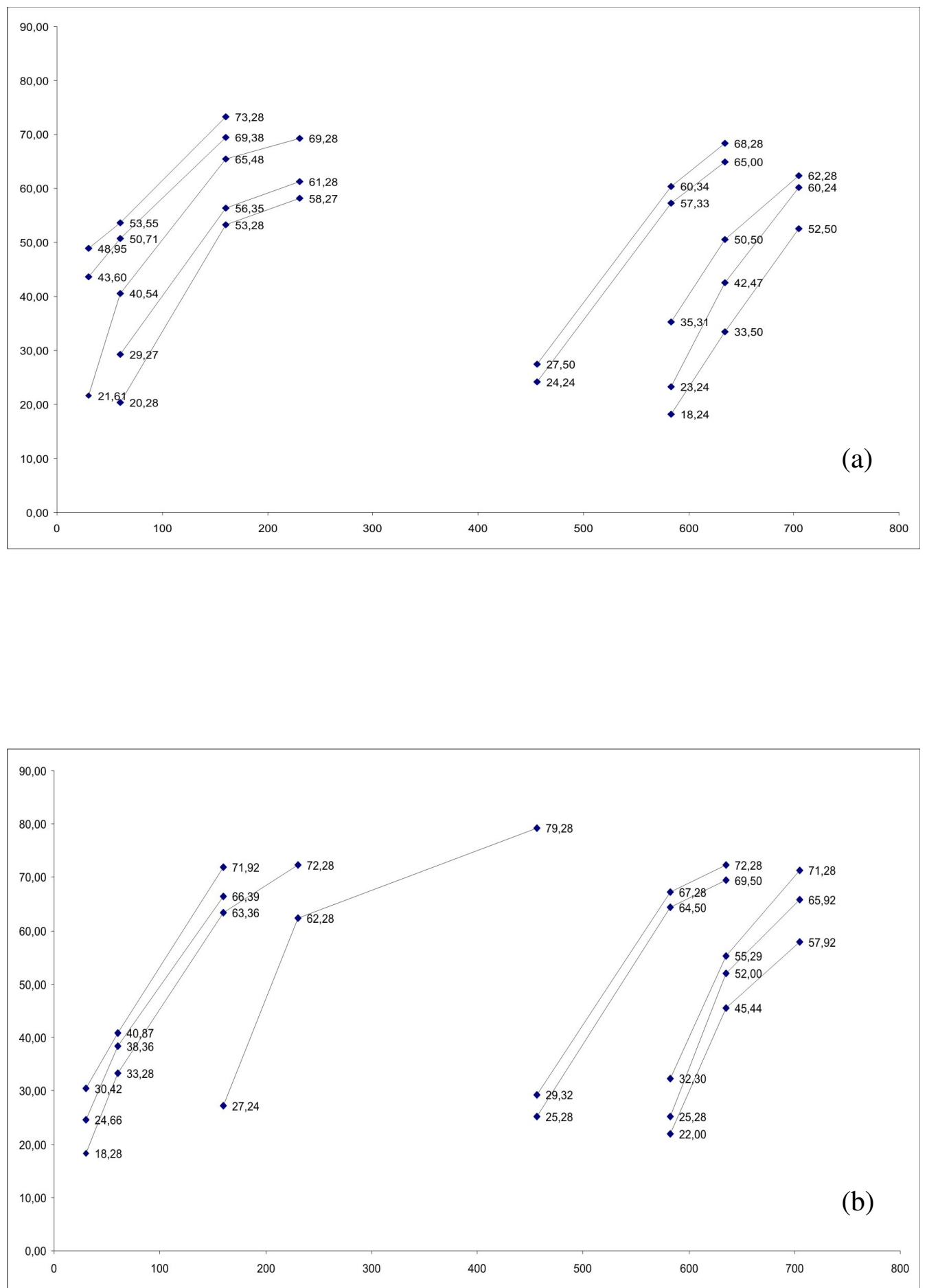

Figura 3. Gráfico com os pontos escolhidos representando as coortes utilizadas na análise de crescimento de machos (a) e fêmeas (b) de Portunus spinicarpus coletados no Litoral Norte de Santa Catarina.

A taxa de crescimento das fêmeas $(k=$ $0,0055 / \mathrm{dia})$ foi um pouco maior que a dos machos $(\mathrm{k}$ $=0,0052 /$ dia) não sendo significativamente diferente $(p>0,05)$, as fêmeas atingiram uma largura de carapaça assintótica $(L C \infty=103,28)$ maior que a dos machos $\left(\mathrm{LC}_{\infty}=92,00\right)$ (Figura 4). 


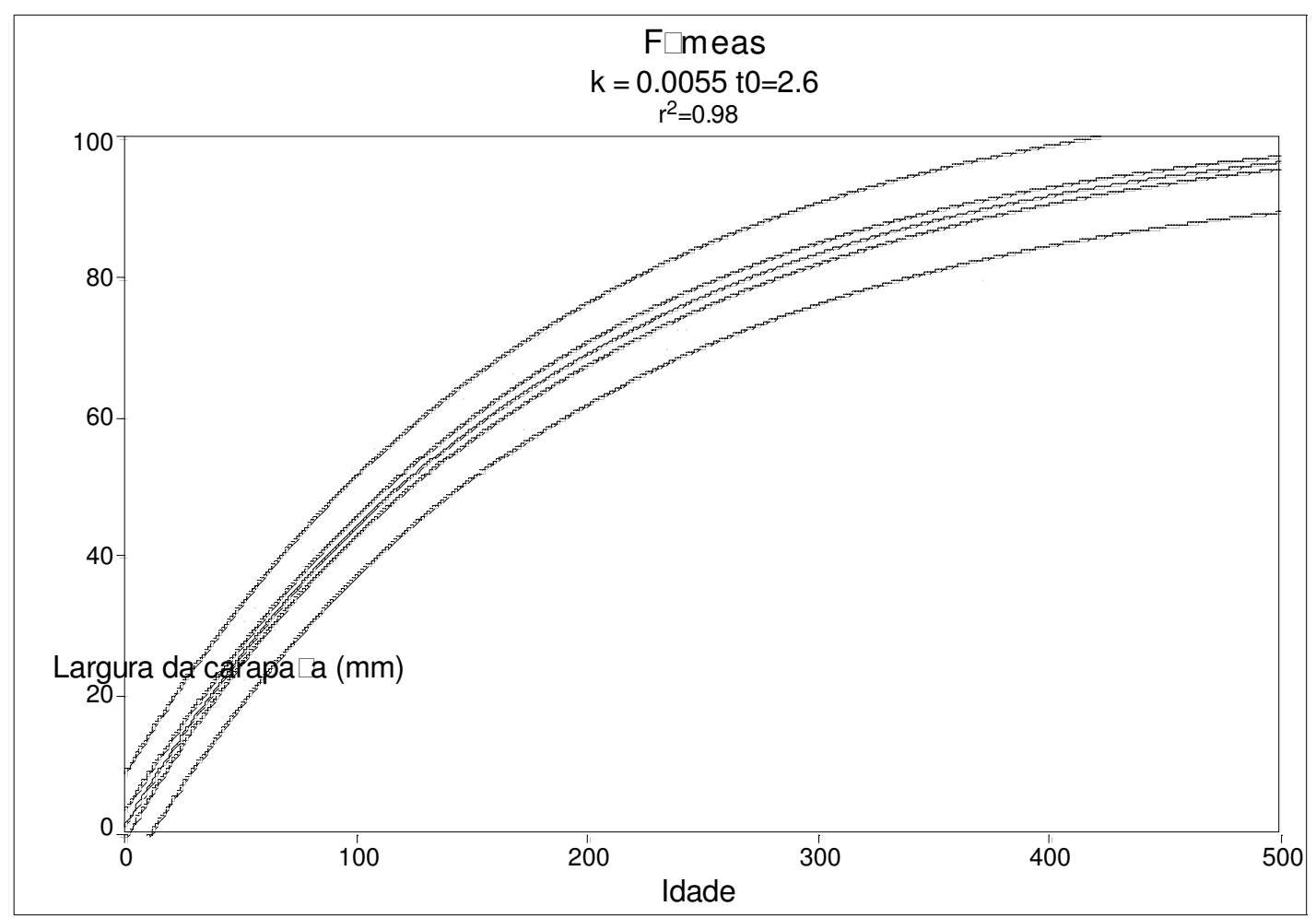

Figura 4. Curva de crescimento e parâmetros da equação de von Bertalanffy estimados para machos (a) e fêmeas (b) de Portunus spinicarpus coletados no Litoral Norte de Santa Catarina, Brasil, durante o período de estudo. A linha central representa a média, enquanto as linhas externas são os intervalos de predição (95\%) e as internas são os intervalos de confiança estimados (95\%).

A longevidade dos machos foi estimada em 2,4 anos enquanto as fêmeas tiveram uma longevidade um pouco menor, estimada em 2,3 anos.

O teste $\mathrm{F}$ para comparação entre as curvas de crescimento de machos e de fêmeas (Cerrato, 1990) mostrou diferença significativa (Fcalculado $=66,845$; Ftabelado $=3,120, p<0,05)$, evidenciando dimorfismo sexual para a espécie em relação ao tamanho dos indivíduos. Isso se deve ao tamanho máximo $\left(\mathrm{LC}^{\infty}\right)$ maior alcançado pelas fêmeas em relação ao dos machos.

Os machos tiveram peso variando de 0,21 a $42,18 \mathrm{~g}$ enquanto as fêmeas variaram de 0,26 a 52,93 $\mathrm{g}$ e as fêmeas ovígeras de 1,69 a 41,65 g.
A relação peso e largura de carapaça para fêmeas foi isométrica com tendência a alométrica positiva enquanto a dos machos é alométrica negativa para indivíduos menores que $60,0 \mathrm{~mm}$ passando a alométrica positiva para indivíduos com $60,0 \mathrm{~mm}$ ou mais de largura de carapaça (Figuras $5 \mathrm{e}$ 6),. A relação peso em função da largura de carapaça (LC) para machos e fêmeas mostrou-se significativamente diferente $(\mathrm{F}$ calculado $=74,15 ; \mathrm{F}$ tabelado $=3,84 ; p<0,05)$. 


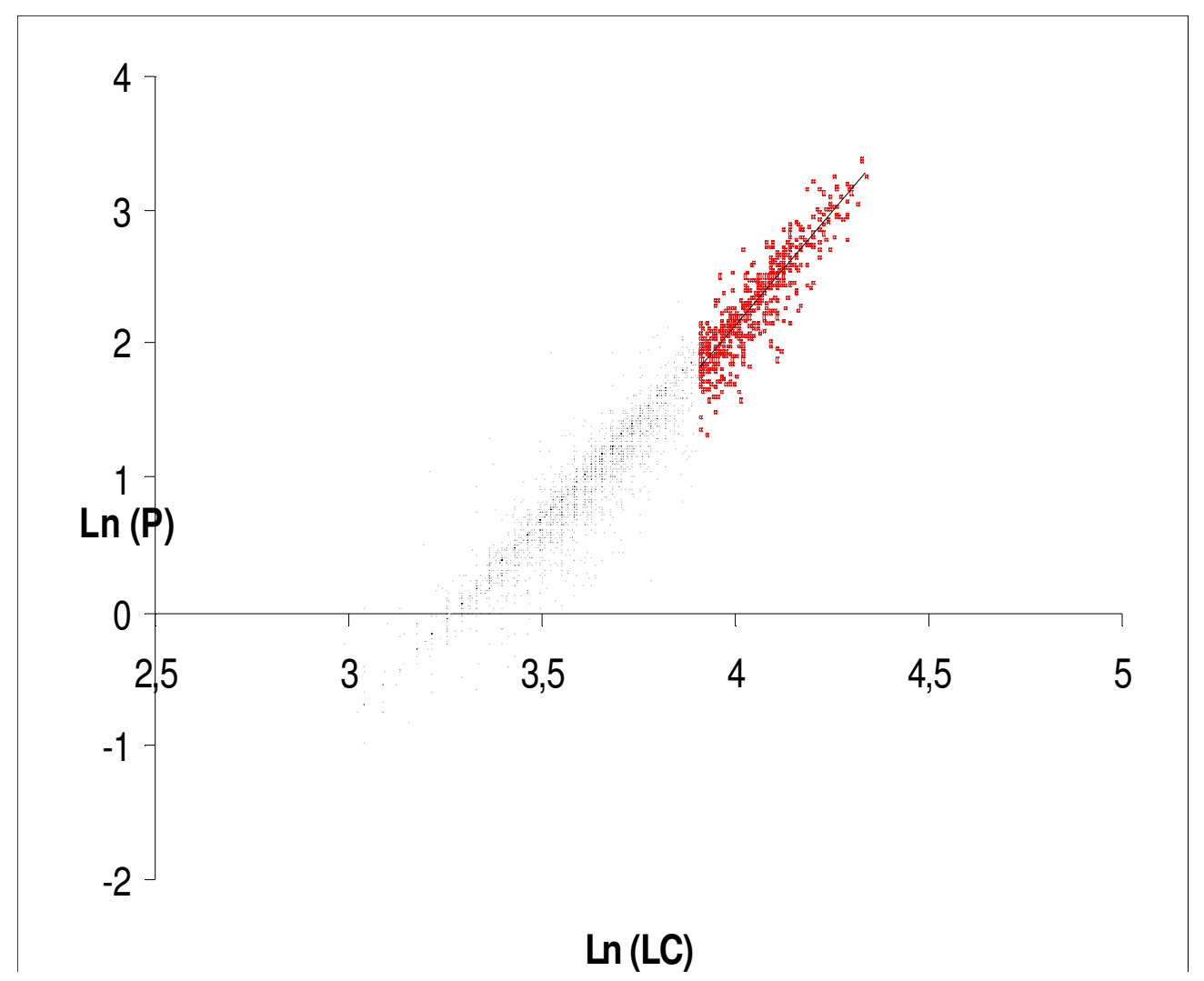

Figura 5. Transformação logarítmica da relação entre peso $L n(P)$ e largura de carapaça Ln (LC) para machos de Portunus spinicarpus. No gráfico, a cor vermelha corresponde animais com crescimento alométrico positivo.

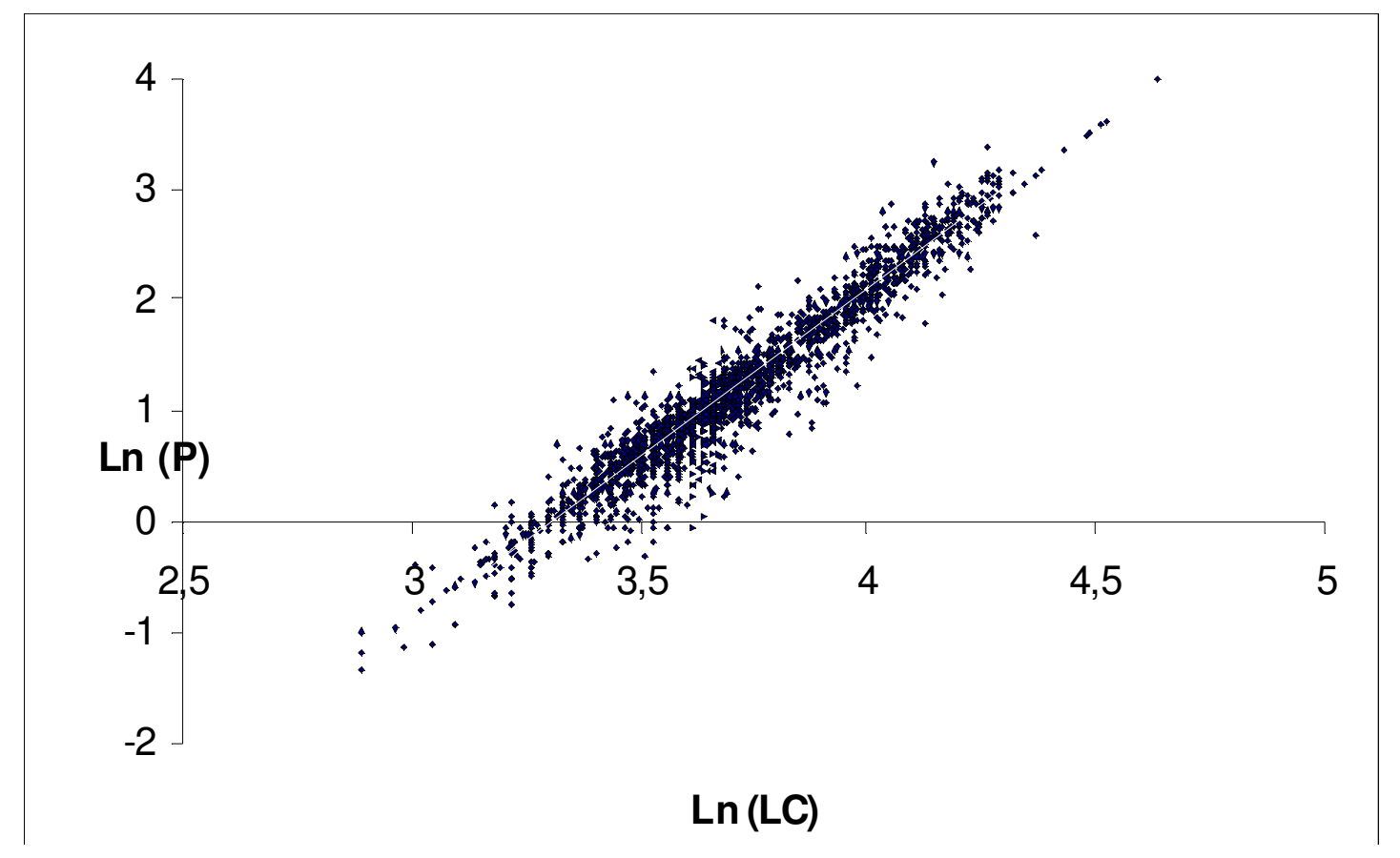

Figura 6. Transformação logarítmica da relação entre peso $L n(P)$ e largura de carapaça $L n(L C)$ para fêmeas de Portunus spinicarpus. 
Quando analisado o crescimento alométrico de machos em diferentes classes de tamanho foi constatada diferença significativa (para intervalos de confiança de valores de "b") entre animais com tamanho menor que 60,0 $\mathrm{mm}(\mathrm{b}=2,86 \pm 0,05)$ e aqueles com tamanho igual ou maior que $60,0 \mathrm{~mm}(\mathrm{~b}=3,37 \pm 0,10)$. Essa classe de tamanho em que o crescimento muda significativamente foi definida a partir do ajuste dos resíduos no programa Table Curve a partir dos dados logaritmizados. Considerando os valores de "b", animais com LC até 60,0 mm têm crescimento alométrico negativo, enquanto os indivíduos com LC igual ou maior que $60,0 \mathrm{~mm}$ crescem com alometria positiva.

As correlações, expressas pelos valores de $R_{2}$, entre LC e P para esta espécie mostraram-se diferentes tanto entre os sexos como entre as classes de tamanho observadas para machos (Tabela 3).

Tabela 3. Equações logarítmicas da relação peso (P) em função da largura de carapaça (LC), com os respectivos coeficientes de determinação $\left(r^{2}\right)$ e índices de alometria.

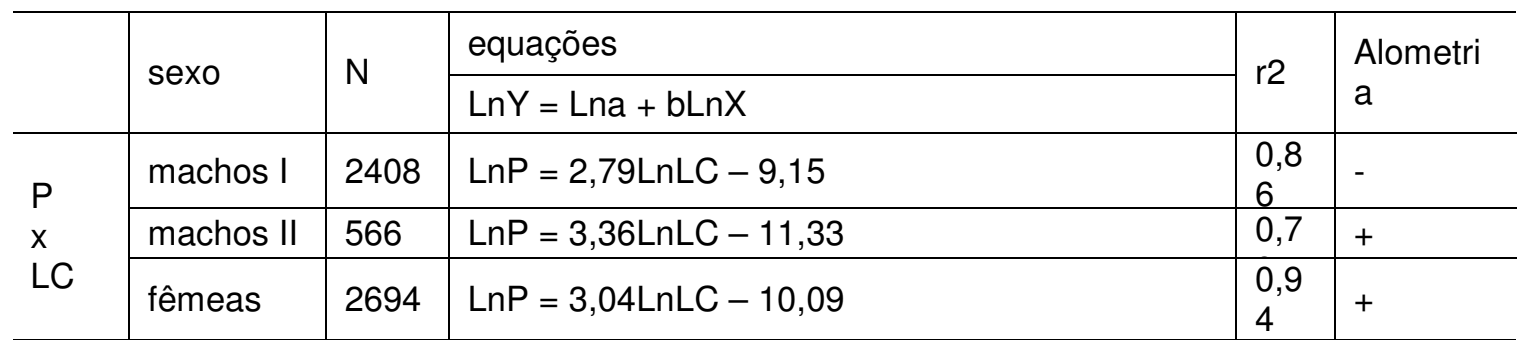

\section{DISCUSSÃO}

Diferenças nas características populacionais têm sido observas não somente entre diferentes espécies, como também entre populações de uma mesma espécie em distintas regiões geográficas. $O$ tamanho assintótico observado para $P$. spinicarpus no presente trabalho difere do padrão observado para os

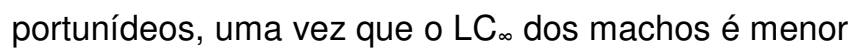
do que o das fêmeas.

Corbi-Corrêa \& Fransozo (2002) realizaram análises de relações entre dimensões do corpo de $P$. spinicarpus e estimaram crescimento alométrico, não foi aplicado o modelo de Von Bertalanffy para determinar a curva de crescimento e idade nessa espécie.

A partir da curva de crescimento de $P$. spinicarpus calculou-se a idade em um determinado tamanho. Dessa forma, estima-se que o tamanho de primeira maturação para fêmeas corresponde a 134 dias (aproximadamente 4,5 meses), enquanto os machos maturam com 229 dias (aproximadamente 7,6 meses).

A determinação da idade bem como os demais parâmetros da curva de crescimento de uma espécie tem sido considerada necessária para quase todos os estudos de dinâmica populacional (Lee \& Hsu, 2003).
Além disso, estudos sobre o crescimento de caranguejos criados em cativeiro e experimentos de marcação e recaptura são necessários para se desenvolver métodos de determinação e validação de idade (Lee \& Hsu, 2003).

Embora o tempo máximo de vida de $P$. spinicarpus tenha sido estimado em 2,4 anos para machos e 2,3 anos para fêmeas, apenas uma pequena porção da população irá atingir essa idade, como resultado da predação e mortalidade natural. Durante o período de coleta, observou-se um declínio na idade da população através da distribuição de freqüência por classes de tamanho para cada amostra. Nas primeiras amostras foram capturados indivíduos em mais classes de tamanho do que nas últimas. A ultima amostragem apresentou indivíduos concentrados nas classes de tamanho intermediários e menores.

Uma comparação entre o crescimento de machos e de fêmeas poderia demonstrar que as fêmeas crescem ligeiramente mais rápido que os machos, atingindo tamanhos maiores em menos tempo. No entanto, essa diferença não é significativa uma vez que não houve diferenças significativas entre as taxas de crescimento.

A relação peso-largura da carapaça tem sido utilizada para facilitar a estimativa do peso de um 
exemplar através de sua largura e determinar o tipo de crescimento das espécies (Branco et al., 2002).

O parâmetro "b" da relação peso-largura de carapaça pode ser utilizado para detectar variações sazonais no crescimento das espécies, podendo variar de acordo com a disponibilidade de alimento e com o estágio de desenvolvimento reprodutivo da população (King, 2003). É o que foi verificado entre os machos jovens e adultos no presente trabalho.

Não foi observado para o crescimento relativo de fêmeas de $P$. spinicarpus diferenças significativas entre classes de tamanho, o que permite afirmar que a relação peso-largura estimada para fêmeas aplicase a qualquer fase de desenvolvimento. No entanto, isso não se aplica aos machos. Para machos foi observada uma mudança no padrão de alometria com aumento do LC. Dessa forma, pode-se sugerir que o tamanho médio de primeira maturação para machos de $P$. spinicarpus seja estimado entre 60,0 e $70,0 \mathrm{~mm}$ (ponto de inflexão estabelecido em $64,0 \mathrm{~mm}$ ) uma vez que a partir dessa classe de tamanho há uma mudança marcada no tipo de crescimento. Essa mudança, provavelmente, é resultado da ocorrência de uma muda de puberdade, em que os machos passam a crescer mais em peso como resultado do aumento do tamanho das quelas ou pelo incremento no peso das gônadas. Essa forma de crescimento, em que os machos tendem a crescer mais em peso do que na largura, pode estar relacionada com a sua biologia como foi observado para outros siris (Mantelatto \& Fransozo, 1996 e Olmi \& Bishop, 1983). Os Brachyura machos, de um modo geral, precisam maximizar o seu tamanho para poder alcançar sucesso em estratégias reprodutivas, principalmente, através do desenvolvimento de seus quelípodos. Estes são estruturas utilizadas na defesa de território e manipulação de fêmeas durante a cópula (Santos et al., 1995; Mantelatto \& Martinelli, 1999). Assim, por crescerem mais nos machos, o peso dos quelípodos passa a contribuir, significativamente, para essa diferença no peso de acordo com o sexo. CorbiCorrêa \& Fransozo (2002) constataram que a relação comprimento do propodo quelar e largura de carapaça em machos apresenta alometria positiva e é um bom indicador para tamanho de primeira maturação, uma vez que cresce bem mais nos machos do que nas fêmeas.
Outro fator que pode contribuir para essa diferença no crescimento dos machos em relação as fêmeas é que elas tendem a investir mais energia na reprodução do que no crescimento somático (Mantelatto \& Martinelli, 1999). Esse dimorfismo na relação peso-largura da carapaça condiz com o observado para $P$. spinicarpus já que machos e fêmeas têm crescimento alométrico significativamente diferente.

De acordo com Baptista-Metri et al (2005) a época, local e características ambientais podem propiciar variações no tipo de crescimento dos siris. Dessa forma, se constatam variações no tipo de crescimento dentro de uma mesma espécie. Por exemplo, em Callinectes ornatus foi observado crescimento alométrico positivo tanto para machos como para fêmeas (Baptista et al., 2003; Branco \& Fracasso, 2004; Mantelatto \& Fransozo, 1996) ou alométrico positivo para machos e alométrico negativo para fêmeas (Branco \& Lunardon-Branco, 1993).

Variações intra-específicas dentro de Portunidae também são comuns. Para $P$. spinimanus não encontraram diferenças no crescimento de machos jovens e adultos, sendo que a mesma equação descreve bem a relação peso-largura da carapaça Santos et al. (1995). Os mesmos autores constataram que tanto machos como fêmeas crescem de forma alométrica positiva. No entanto, fêmeas de Arenaeus cribrarius têm crescimento alométrico negativo enquanto machos têm crescimento alométrico positivo (Ávila \& Branco, 1996).

Outra forma de abordar o crescimento relativo em Portunidae e estabelecer tamanho de primeira maturação por mudanças no grau de alometria tem sido o uso de outras relações morfométricas (largura e comprimento do quelípodo, largura do abdômen, largura e comprimento da carapaça). Normalmente nesses trabalhos, a largura da carapaça é considerada a variável independente. No Brasil, Pinheiro \& Fransozo (1993) realizaram esse tipo de trabalho para Arenaeus cribrarius e Santos et al (1995) para Portunus spinimanus. Corbi-Corrêa \& Fransozo (2002) utilizaram as mesmas relações para estabelecer um tamanho de primeira maturação para P. spinicarpus estimado em 27,15 mm LC para fêmeas e 27,30 mm LC para machos. Esses valores, 
provavelmente diferem do obtido neste trabalho pelas seguintes características: metodologia utilizada, em que não foi feita relação peso-largura de carapaça; as medidas foram tomadas na base dos espinhos e o maior indivíduo capturado por esses autores (LC = $52,00 \mathrm{~mm}$ ) era menor que o maior capturado em Santa Catarina.

Os machos da espécie em estudo seguem o padrão de crescimento geral descrito por Hartnoll (1978): existem duas fases claramente diferenciadas separadas pela muda de puberdade.

Sukumaran \& Neelakantan

estabeleceram que os machos de $P$. pelagicus e $P$. sanguinolentus são mais pesados do que as fêmeas numa mesma classe de largura e comprimento de carapaça. No entanto, essas relações em $P$. pelagicus não são significativamente diferentes entre os sexos.

O Código de Conduta para a Pesca Responsável (FAO, 1995) prevê que não somente as

\section{AGRADECIMENTOS}

Os autores agradecem à equipe do CEPSUL/IBAMA por permitir a participação do Laboratório de Crustáceos Decápodes

\section{REFERÊNCIAS BIBLIOGRÁFICAS}

AVILA, MG \& JO BRANCO 1996. Aspectos bioecológicos de Arenaeus cribarius (Lamarck) (Decapoda, Portunidae) da Praia da Barra da Lagoa, Florianópolis, Santa Catarina, Brasil. Rev. Bras. Zool.13(1): 165-174.

BAPTISTA, C, MAA PINHEIRO, A BLANKENSTEYN \& CA BORZONE 2003. Estrutura populacional de Callinectes ornatus Ordway (Crustacea, Portunidae) no Balneário Shangri-Lá, Pontal do Paraná, Brasil. Rev. Bras. Zool. 20(4): p. 661 - 666.

BAPTISTA-METRI, C, MAA PINHEIRO, A BLANKENSTEYN \& CA BORZONE 2005. Biologia populacional e reprodutiva de Callinectes danae Smith (Crustacea, Portunidae), no Balneário Shangri-lá, Pontal do Paraná, Paraná, Brasil. Rev. Bras. Zool. 22(2): 446-453.

BOOKHOUT, CG \& JD COSTLOW 1974. Larval development of Portunus spinicarpus Reared in Laboratory. Bull. of Mar. Sci. 24(1): 20-51.

BRAGA, A.A.; FRANSOZO,A.; BERTINI, G. \& FUMIS, P.B. 2005 Composição e abundância dos caranguejos (Decapoda, Brachyura) nas regiões de Ubatuba e Caraguatatuba, litoral norte paulista, Brasil. Biota Neotrop. Jul/Dez 2005, vol. 5, no.2. Disponível em: <http://www.biotaneotropica.org.br/v5n2/pt/abstract?article+BN0020 5022005 > Acesso em: 6 dez.2005 .ISSN 1676-060 espécies alvo de uma pescaria devem ser preservadas, mas também todas aquelas que compõem o ecossistema ou que sejam dependentes delas ou ainda que estejam a elas associadas. Sendo Portunus spinicarpus pertencente à fauna acompanhante da pesca do camarão na região Sudeste/Sul do Brasil, a realização de estudos sobre a biologia da espécie torna-se importante para garantir sua preservação. A espécie é muito abundante na costa brasileira e sua importância está principalmente relacionada com o seu papel ecológico (Braga et al., 2005; Dodril \& Manooch, 1993; Klein et al, 2001; Léo \& Pires-Vanin, 2006; Mantellato et al.,2003; Muto et al., 2001; Pires, 1992; Vaske \& Castello, 1998). Assim, espera-se que compreendendo melhor a biologia desta espécie, leve-se em consideração sua ecologia na elaboração dos planos de manejo da pesca do camarão.

(LCD) da Fundação Universidade do Rio Grande (FURG) no Projeto Camarões da Baía da Babitonga (CAMBA) e pela publicação dos dados obtidos no referido projeto.

BRANCO, JO \& HAA FRACASSO 2004. Biologia populacional de Callinectes ornatus (Ordway) na Armação do Itacoroy, Penha, Santa Catarina, Brasil. Rev. Bras. Zool. 21(1): p. 91 - 96.

BRANCO, JO \& MJ LUNARDON-BRANCO 1993. "Growth and 1St maturation Size in Callinectes ornatus Ordway, 1863 (Decapoda, Portunidae) from the Matinhos Region, Parana State, Brazil." Arq. Biol. Tecnol.36(3): 497-503.

BRANCO, JO, MJ LUNARDON-BRANCO \& FX SOUTO 2002. "Estrutura populacional de Portunus spinimanus Latreille (Crustacea, Portunidae) na Armação do Itapocoroy, Penha, Santa Catarina, Brasil." Rev. Bras. Zool.19(3): 731-738.

CASTRO FILHO, BM \& LB MIRANDA. 1987. Condições hidrográficas na plataforma continental ao largo de Ubatuba: variações sazonais e em média escala. Bol. Inst. Oceanogr., 35 (2): 135-151.

CERRATO, RM. 1990. Interpretable statistical tests for growth comparisons using parameters in the von Bertalanffy equation. Can. J. Fish. Aquat. Sci., 47: 1416-1426

CORBI-CORRÊA, E \& A FRANSOZO 2002. Growth patterns of Portunus spinicarpus (Stimpson, 1871) (Decapoda, Portunoidea) from Ubatuba region (SP), Brasil. Nauplius 10(2): 131-137.

D'INCAO, F \& DB FONSECA. 1999. Performance of the von Bertalanffy growth curve in penaeid shrimp: a critical approach, $p$. 733-737. In: JC Von VAUPEL KLEIN \& FR SCHRAM. (Eds.). 
Proceedings of the Fourth International Crustacean Congress, Rotterdam, 848p.

de LESTANG, S, N HALL, IC \& POTTER. 2003a. Changes in density, age composition, and growth rate of Portunus pelagicus in a large embayment in which fishing pressures and environmental conditions have been altered. J. Crustacean Biol. 23(4): 908-919.

de LESTANG, S, N HALL, IC \& POTTER. 2003b. Reproductive biology of the blue swimmer crab (Portunus pelagicus, Decapoda: Portunidae) in five bodies of water on the west coast of Australia. Fish. Bull. 101(4): 745-757.

DODRILL, J \& AB MANOOCH. 1993. Food and Feeding-Behavior of Adult Snowy Grouper, Epinephelus niveatus (Valenciennes) (Pisces, Serranidae), Collected off the central North-Carolina Coast with ecological notes on major food groups. Brimleyana, 19: 101135

FAO, 1995. Code of Conduct for Responsible Fisheries. FAO, Roma, 45p.

FONSECA, DB. 1998. Kalliapseudes schibartii (Crustácea, Tanaidacea): comparação entre metodologias de análise de crescimento e dinâmica populacional da espécie. Fundação Universidade Federal do Rio Grande: Rio Grande Dissertação de Mestrado, $103 \mathrm{p}$.

HARTNOL, RG. 1982. Growth. In: ABELE, LG. ed. The Biology of Crustacea: Embryology, Morphology and Genetics. New York, Academic.v.2,p. 11-196.

HARTNOLL, RG 1978. The Determination of relative growth in Crustacea. Crustaceana 34(3): 281-293.

JOSILEEN, J \& NG MENON 2005. Growth of the blue swimmer crab, Portunus pelagicus (Linnaeus, 1758) (Decapoda, Brachyura) in captivity. Crustaceana 78: 1-18.

KING, M. 2003. Fisheries Biology, assessment and management. Oxford, Fishing New Books. 341p.

KLEIN, JA, CA BORZONE \& PR PEZZUTO. 2001. A Macro e Megafauna Bêntica Associada aos Bancos da Vieira Euvola ziczac (Mollusca: Bivalvia) no Litoral Sul do Brasil. Atlântica. 23: 17-26

LEE, HH \& CC HSU 2003. Population biology of the swimming crab Portunus sanguinolentus in the waters off northern Taiwan. J. Crustacean Biol. 23(3): 691-699.

LÉO, FCD \& AMS PIRES-VANIN 2006. Benthic megafauna communities under the influence of the South Atlantic Central Water intrusion onto the Brazilian SE shelf: A comparison between an upwelling and a non-upwelling ecosystem. J. Mar. Syst. 60: 268 284.

MANTELATTO, FLM \& A FRANSOZO 1996. Size at sexual maturity in Callinectes ornatus (BRACHYURA, PORTUNIDAE) from the Ubatuba region (SP) Brazil. Nauplius 4: 29-38.

MANTELATTO, FLM \& A FRANSOZO. 1992. Relação peso/largura da carapaça no caranguejo Hepatus pudibundus (Herbst,
1785)(Crustacea, Decapoda, Calappidae) na região de Ubatuba, SP, Brasil. Arq. Biol. Tecnol., 35 (4): 719-724.

MANTELATTO, FLM \& JM MARTINELLI 1999. Carapace widthweight relationships of Callinectes ornatus (Brachyura, Portunidae) from Ubatuba Bay, Brazil. Iheringia, Série Zoologia, Porto Alegre, 87: 111-116.

MELO, GAS. 1996. Manual de identificação dos Brachyura (caranguejos e siris) do litoral brasileiro. São Paulo: Plêiade/FAPESP. 604 pp.

MUTO, EY, LSH SOARES \& R GOITEIN 2001. Food resource utilization of the skates Rioraja agassizii (Müller \& Henle, 1841) and Psammobatis extenta (Garman, 1913) on the continental shelf off Ubatuba, South-Eastern Brazil. Rev. Bras. Biol. 61(2): 217-238.

OLMI, EJ \& JM BISHOP 1983. Variations in total width-weight relationships of blue crabs, Callinectes sapidus, in relation to sex, maturity, molt stage, and carapace form. J. Crust. Biol. 3(4): 575581.

PINHEIRO, MAA \& A FRANSOZO 1993. Relative growth of the speckled swimming crab Arenaeus cribrarius (Lamarck, 1818) (Brachyura, Portunidae), near Ubatuba, State of Sao Paulo, Brazil. Crustaceana 65(3): 377-389.

PIRES, AMS 1992. Structure and dynamics of benthic megafauna on the continental-shelf offshore of Ubatuba, Southeastern Brazil. Mar. Ecol. Prog. Ser. 86(1): 63-76.

POTTER, IC, PJ CHRYSTAL \& NR LONERAGAN 1983. The Biology of the blue manna crab Portunus pelagicus in an Australian Estuary. Mar. Biol. 78(1): 75-85.

SANTOS, S, ML NEGREIROS-FRANSOZO \& CR PADOVANI 1995. Relação do peso do corpo em função da largura da carapaça do siri candeias Portunus spinimanus Latreille, 1819 (Decapoda, Portunidae). Arq. de Biol. e Tecnol. 38(3): 715-724.

SUKUMARAN, KK \& B NEELAKANTAN 1997. Sex ratio, fecundity and reproductive potential in two marine portunid crabs, Portunus (Portunus) sanguinolentus (Herbst) and Portunus (Portunus) pelagicus (Linnaeus) along the Karnataka coast. Indian J. Mar. Sci. 26(1): 43-48.

SUMPTON, WD, MA POTTER \& GS SMITH 1994. Reproduction and growth of the commercial sand crab, Portunus pelagicus (L.) in Moreton Bay, Queensland. Asian Fish. Sci. 7: 103 - 113.

VASKE JR., T \& JP CASTELLO 1998. Conteúdo estomacal da albacora-laje, Thunnus albacares, durante o inverno e primavera no sul do Brasil. Rev. Bras. Zool. 58(4): 639-647.

Recebido: 03/06/2007

Aceito: $03 / 08 / 2010$ 
